\title{
DEVELOPMENT OF A WATER MONITORING SYSTEM BASED ON INTEGRATED POLYMER MICROFLUIDICS
}

\author{
L. Zhu ${ }^{1}$, D. Meier ${ }^{2}$, Z. Boger ${ }^{2,3}$, C. Montgomery ${ }^{2}$, S. Semancik ${ }^{2}$, D.L. DeVoe ${ }^{1}$ \\ ${ }^{1}$ Department of Mechanical Engineering, University of Maryland, College Park, MD 20742 USA \\ ${ }^{2}$ National Institute of Standards and Technology, Gaithersburg, MD 20899 USA \\ ${ }^{3}$ OPTIMAL - Industrial Neural Systems Ltd., Be'er Sheva, 84243 ISRAEL
}

\begin{abstract}
A polymer microfluidic system for monitoring of volatile organic compounds diluted in water is presented. The sensor platform employs silicon-based microhotplate gas sensors as active detection elements, using a silicon-in-plastic microfabrication technology to enable integration of individual sensor chips into a larger polymer microfluidic substrate. The fabrication process provides seamless fluidic and electrical interconnects between the silicon and polymer substrates. The chemical monitoring system is designed to sample a water source, extract solvent present within the aqueous sample into the vapor phase, and direct the solvent vapor past the integrated gas sensor for analysis. Design, fabrication, and characterization of a prototype system is described, and results from illustrative measurements performed using methanol, toluene, and 1,2-dichloroethane in water are presented.
\end{abstract}

\section{INTRODUCTION}

There is growing concern regarding the quality of water supplies, which are susceptible to both natural and man-made chemical contamination. Contamination by volatile organic compounds (VOCs) is of particular concern because many VOCs produce serious health effects, such as liver or nervous system problems, reproductive difficulties, and increased risk of cancer [1]. The U.S. Environmental Protection Agency (EPA) currently sets maximum contaminant levels for safe drinking water on $53 \mathrm{VOC}$ contaminants. A variety of detection methods have been employed for water analysis, such as LC/MS and GC/MS [2]. However, these methods generally rely on labor-intensive sample collection and laboratory-based analysis, which limit the quality, quantity, and frequency of data collected. These factors have led to a need to develop new technology for in-situ VOC monitoring system which is sensitive, selective, and compact, and which involves low power consumption for use in long-term monitoring applications.

Reports from the U.S. EPA have suggested that polymerabsorption and metal-oxide-semiconductor sensors are viable candidates for use in such a sensing system based on their simplicity and robustness [3], and several efforts have addressed the development of such sensing systems. For example, researchers at Sandia National Laboratories have developed an in-situ polymer-based chemiresistor sensing system for real-time detection of VOCs in soil and groundwater [4]. Ho et al. reported a surface acoustic wave sensor for in-situ monitoring of VOCs which consists of a chemically adsorbent polymer film on a piezoelectric substrate [5], and a microscale gas chromatography system for biomedical and environmental monitoring applications was recently reported [6]. However, these devices rely on passive transport of gas from a water source, and do not support higher levels of integration for water delivery or vapor pressure control. Furthermore, the performance of these devices was impacted by environmental temperature fluctuations.
In the present work, we report the application of silicon-inplastic microfabrication technology to the development of an integrated microfluidic gas sensor for the monitoring of VOCs in water supplies. The silicon-in-plastic microfabrication method provides the ability to use no more than the necessary high cost silicon area, and thereby offers the potential to substantially reduce overall system fabrication costs. The microfluidic system provides the required fluid routing, while offering several advantages for efficient solvent extraction such as large specific interface area, large ratio between interface area and channel volume, and short diffusion distance and time [7]. Furthermore, microfluidic systems can readily support integrated microheaters to control the liquid and vapor temperature, and thereby enhance the solvent concentration delivered to the gas sensor chip.

\section{FABRICATION}

The fabrication process developed for integration of bare gas sensor chips into a polymer microfluidic substrate is depicted in Figure 1. A round hole $3 \mathrm{~mm}$ in diameter is first milled through a $1.5 \mathrm{~mm}$ thick polycarbonate (PC) substrate. A microhotplate gas sensor chip is next placed face-down on a flat layer of partially cured polydimethylsiloxane (PDMS, Sylgard 184; Dow Corning, Midland, MI). The PC substrate is aligned over the chip and placed onto the PDMS with the chip centered inside the milled hole. The assembly is placed in a vacuum chamber and the milled hole is filled with epoxy (353ND; Epoxy Technology, Billerica, MA). After the epoxy is cured for $12 \mathrm{hr}$ at room temperature, followed by $12 \mathrm{hr}$ at $50{ }^{\circ} \mathrm{C}$, the PDMS layer is removed. The epoxy serves to secure the chip within the PC substrate, while leveling the chip surface to within $1 \mu \mathrm{m}$ of the PC surface. But small gaps can form at the silicon/epoxy/PC interfaces due to thermal mismatch and epoxy shrinkage during curing. These gaps, which are typically on the order of several microns deep, can cause several problems when forming metal interconnects between the PC substrate and on-chip bond pads. To overcome these problems, a $6 \mu \mathrm{m}$ layer of parylene $\mathrm{C}$ is deposited to passivate the sidewalls and bridge small gaps prior to metal deposition. Parylene $\mathrm{C}$ covering the bondpads and microhotplates of the gas sensor is removed by oxygen plasma etching using a $\mathrm{Cr} / \mathrm{Au}$ mask. After the parylene layer is patterned, the mask metals are stripped, and new $\mathrm{Cr}(20 \mathrm{~nm})$ and $\mathrm{Au}(500 \mathrm{~nm})$ layers are deposited and patterned to provide interconnections from the bondpads on the gas sensor to connection pads on the PC substrate. Finally, conductive epoxy was applied at the connection pads to provide electrical access to the system. A photograph of a typical substrate fabricated using this approach is shown in Figure 2.

The integrated sensor array platforms used in this work are 4-element microhotplate gas sensor chips designed by NIST researchers [8] and fabricated at MIT Lincoln Laboratory. Each chip is $1.6 \mathrm{~mm}$ square. After integrating the silicon chip, a $\mathrm{XeF}_{2}$ isotropic silicon etch is used to remove silicon from beneath the hotplate, allowing final release of the suspended microhotplates insitu. After release, semiconducting metal oxides are grown on the 
microhotplate elements to provide the required sensor films by chemical vapor deposition.
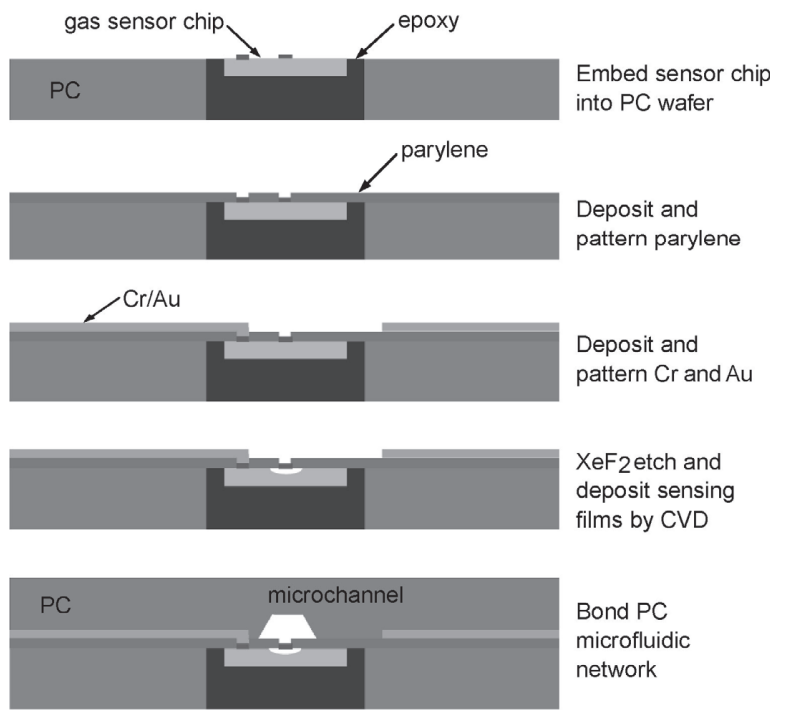

Fig.ur.e 1. Fabrication process for the integrated microfluidic gas sensing system.

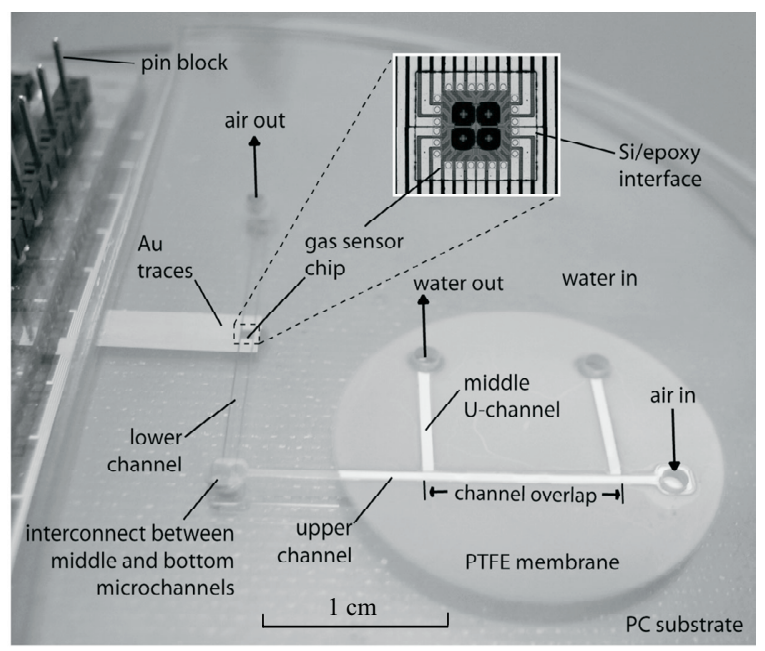

Figure 2. Photograph of a fabricated microfluidic substrate with integrated microhotplate gas sensor chip.

Gas-phase solvent extraction from the aqueous phase is a key requirement for the water monitoring system, since solvent sensing is ultimately performed in the gas-phase. The solvent extraction subsystem was fabricated using polymer microfluidic technology. The design consists of two microchannels separated by a porous hydrophobic membrane. The microchannels are trapezoidal in cross-section and $100 \mu \mathrm{m}$ deep with a width at half-depth of 640 $\mu \mathrm{m}$, and were fabricated by hot embossing using a bulk-etched silicon template. A $50 \mu \mathrm{m}$ thick polytetrafluoroethylene (PTFE) membrane with $70 \%$ porosity and $0.22 \mu \mathrm{m}$ average pore size was thermally bonded between the upper (straight microchannel) and middle (U-shape microchannel) PC layers, creating a porous interface where the channels overlap. Next, a bottom PC layer was bonded to the composite structure using solvent bonding. The bottom PC layer contains a microchannel on the exposed side, which is in fluid connection with the straight channel in the upper PC layer by aligned two $1.5 \mathrm{~mm}$ diameter holes premilled at the end of the middle and bottom microchannels before bonding. This microchannel is used to deliver gas to the microhotplate sensor chip. The 3-layer PC microfluidic assembly was fixed to the bottom substrate containing the silicon sensor chip by mechanical clamping, with the microchannel in the bottom of the 3-layer structure aligned over the sensor elements. In the final assembly, the middle U-shaped channel delivers a continuous stream of water which passes over the middle microchannel with the PTFE membrane preventing the aqueous solution from entering the upper channel. Due to its native vapor pressure, solvent within the water passes through the membrane in the gas phase. A continuous flow of dry air is supplied to one end of the upper channel, which delivers the solvent to the gas sensor through the channels in the upper and bottom PC layers.

\section{VAPOR TRANSPORT MODEL}

In order to optimize the design of the air-liquid two-phase flow system, a model for vapor transport across the porous membrane was employed. TeGrotenhuis et al. previously reported a model for a solvent extraction system having two immiscible fluids flowing through rectangular microchannels separated by a porous plate [9]. This model can be readily modified for the case where the water channel contains a liquid phase, while the membrane and the air channel support a gas phase. As described in the TeGrotenhuis model, the boundary condition governing analyte flux between the liquid phase within the water channel and gas phase within the membrane is given by,

$$
D_{l} \frac{\partial C_{l}}{\partial y}=k\left(C_{m}-m_{a} C_{l}\right)
$$

where $D_{l}$ is the diffusion coefficient of the solvent in liquid, and $C_{l}$ and $C_{m}$ are the solvent concentrations within the liquid and membrane, respectively, at the liquid/membrane interface. The parameter $m_{a}$ is the equilibrium distribution coefficient which is defined by the ratio of the equilibrium analyte concentration in the air channel to analyte concentration in the liquid channel at the liquid/membrane interface. This parameter is equivalent to the Henry's law constant for the solvent. The parameter $k$ is an overall gas phase mass transfer coefficient defined by the mass transfer resistance across the liquid/air interface. For the present case, k was assumed to be $0.1 \mathrm{~cm} / \mathrm{sec}$ based on the mass transfer coefficient of six organic compounds provided by Guo and Roache [10]. Beyond these modifications, the original TeGrotenhuis model was used to predict solvent effluent concentration within the air microchannel.

A numerical simulation of effluent concentration was performed over a range of air and liquid flow rates for the case of methanol. Air and liquid diffusion coefficients of $D_{a}=0.159 \mathrm{~cm}^{2} / \mathrm{s}$ and $D_{l}=1.9 \times 10^{-5} \mathrm{~cm}^{2} / \mathrm{s}[11]$ were used. The diffusion constant for methanol within the membrane, $D_{m}$, was estimated analytically using a model for gas diffusion in porous media [12],

$$
D_{m}=D_{a} \varepsilon^{\frac{4}{3}}
$$

where $\varepsilon$ is the membrane porosity. Using this model with $\varepsilon=0.7$ yields $D_{m}=0.079 \mathrm{~cm}^{2} / \mathrm{s}$. A $1 \mathrm{~cm}$ long, $100 \mu \mathrm{m}$ deep intersection region was defined between the liquid and air microchannels. The results were normalized to the saturated vapor concentration of the liquid analyte as determined from the Henry's law constant for methanol, given on a mole fraction basis as 0.29 at $25{ }^{\circ} \mathrm{C}$ [11]. As can be seen from Figure 3 , the effluent concentration may be increased by raising the liquid flow rate and reducing the air flow rate. This result is reasonable, since higher liquid flow rates will rapidly replenish analyte in the water as gas-phase analyte is delivered across the membrane. Similarly, lower air flow rates will allow gas-phase analyte to accumulate in the microchannel, leading to higher effluent concentrations. 


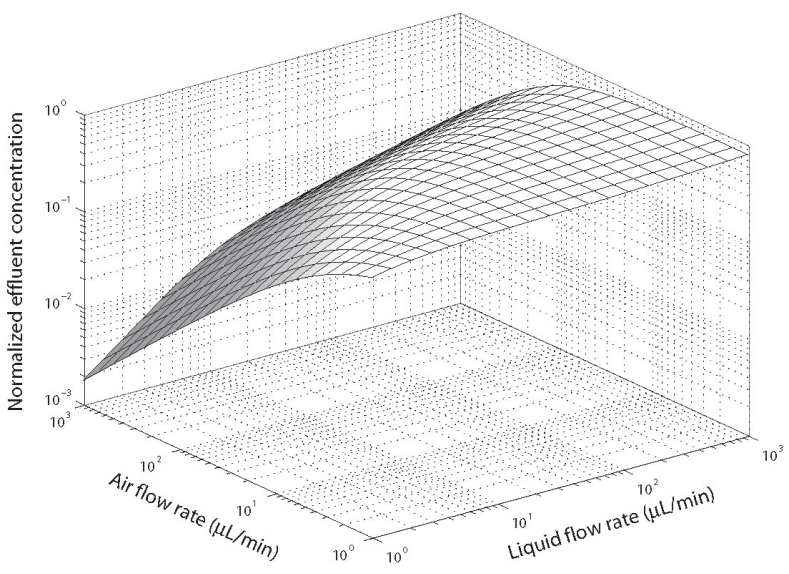

Figure 3. Numerical results of methanol effluent concentration with different air and liquid flow rates.

\section{EXPERIMENTAL RESULTS AND DISCUSSION}

While the equilibrium responses of the metal oxide films have been employed for various detection and monitoring applications, it has been shown that the transient (or kinetic) responses can be particularly useful to distinguish between various analytes. The imposed temperature change in a hotplate element results in a transient response in the sensor film conductance which provides a basis for achieving gas identification as well as measuring concentration by employing a temperature programmed sensing methodology $[13,14]$. The TPS program used in this work alternates the sensor temperature between a ramp value (from 60 ${ }^{\circ} \mathrm{C}$ to $480{ }^{\circ} \mathrm{C}$ in 29 equal steps) and a base value $\left(200{ }^{\circ} \mathrm{C}\right)$ every $500 \mathrm{~ms}$. In this way, 58 data points (conductance of the sensing film, 29 corresponding to the ramp temperature and 29 to the base temperature) are collected for each sensor in $14.5 \mathrm{sec}$. After $5 \mathrm{sec}$ at the base temperature, this temperature sequence is repeated throughout the analyte delivery program.

A microfluidic water monitoring system with an integrated microhotplate gas sensor was fabricated with a $1 \mathrm{~cm}$ overlap region between the liquid and air channels to evaluate the performance of the water monitoring sensor platform. A syringe pump was used to deliver a precise flow of water/solvent mixture in the liquid channel, and a mass flow controller was used to deliver dry air flow in the gas channel. A laptop computer provided temperature control and monitoring of conductometric response for the integrated gas sensor elements. Based on the model results, the flow rate of dry air was set to the minimum value supported by the mass flow controller of $500 \mu \mathrm{L} / \mathrm{min}$, and the flow rate of liquid was set to a relatively high value of $20 \mu \mathrm{L} / \mathrm{min}$. While higher liquid flow rates are desirable to enhance the solvent concentration in the air channel, flow rates above $20 \mu \mathrm{L} / \mathrm{min}$ were often observed to produce leakage in the microfluidic system. At the start of each experiment, air and liquid were pumped to their channels for several minutes to stabilize the air/liquid interface. After stabilization, the TPS program was started. Measurements were performed using 3 different VOC analytes: methanol $(0,1,10$, and $100 \mathrm{ppm})$, toluene $(0,10$, and $100 \mathrm{ppm})$, and 1,2-dichloroethane $(0$, $10,100$, and $1000 \mathrm{ppm})$. Measurements of sensor response for toluene and 1,2-dichloroethane were not performed at $1 \mathrm{ppm}$ due to the relatively low signal observed at $10 \mathrm{ppm}$. All reagents were procured from Fisher Scientific (Fair Lawn, New Jersey).

Conductance measurements over two TPS cycles from a single microhotplate for different concentrations of methanol are shown in Figure 4. This data has been filtered using a running normalization approach, in which the initial conductance measurement for each cycle is normalized by the final value measured in the previous cycle, thereby limiting the effects of baseline signal drift. As can be seen from Figure 4, the characteristics of the TPS response vary as a function of concentration, and these features may be used as signatures for analyte identification and quantification. Artificial neural networks (ANNs) offer an efficient and rapid means of recognition and classification of analytical data, and have recently been applied to the problem of analyte identification and quantification from microhotplate gas sensor data [15].

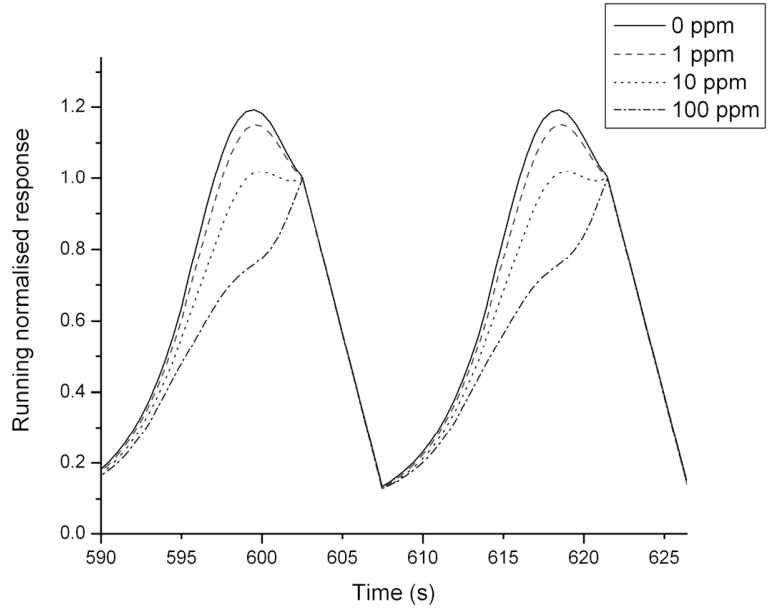

Figure 4. Example response over two TPS cycles to methanol at constant concentration levels from 0 ppm to $100 \mathrm{ppm}$.

In this work, the Guterman-Boger ANN modeling approach was applied to the methanol, toluene, and 1,2-dichloroethane TPS data measured using the integrated microfluidic platform. The data from a minimum of 25 TPS cycles for each analyte and analyte concentration were presented to the ANN as a 116-input vector (29 temperature steps on four sensors). The ANN was trained using $2 / 3$ of the sensor data selected randomly, and the remaining $1 / 3$ of the data was reserved as a validation data set. The random training and validation selection measures the consistency of the data within each analyte concentration value test. The resulting ANN model was applied to both the training and validation data sets. The estimated concentrations for each analyte are shown in Figure 5(a)(c). Because the sensing films generally take about $1 \mathrm{~min}$ to reach equilibrium after being exposed to a new analyte concentration, the first 3 TPS cycles were removed from the these plots.

The average estimated concentrations are given in Figure 6 as a function of the known concentrations. The error bars in this figure represent $+/-\sigma$ for the estimations at each concentration level. Due to the logarithmic axis, any non-positive concentration estimates are not shown in this figure. A summary of the estimated concentration error and standard deviation relative to the known concentrations is provided in Table 1. Predictions for methanol concentrations down to $10 \mathrm{ppm}$ are quite good, with the concentration underestimated by $8 \%$. When reducing the methanol concentration to $1 \mathrm{ppm}$, the estimation error increases to $40 \%$, but with minimum deviation in the estimations. In contrast, the toluene estimation error is $-3.6 \%$ at $10 \mathrm{ppm}$, but with a large standard deviation equal to $28.5 \%$ of the actual concentration level. The estimation error for 1,2-dichloroethane is even larger, with $-62.2 \%$ error at $10 \mathrm{ppm}$ and high variance in the data. Based on the measured data, approximate detection limits for the fabricated system are $1 \mathrm{ppm}$ for methanol, $10 \mathrm{ppm}$ for toluene, and $100 \mathrm{ppm}$ for 1,2-dichloroethane. 

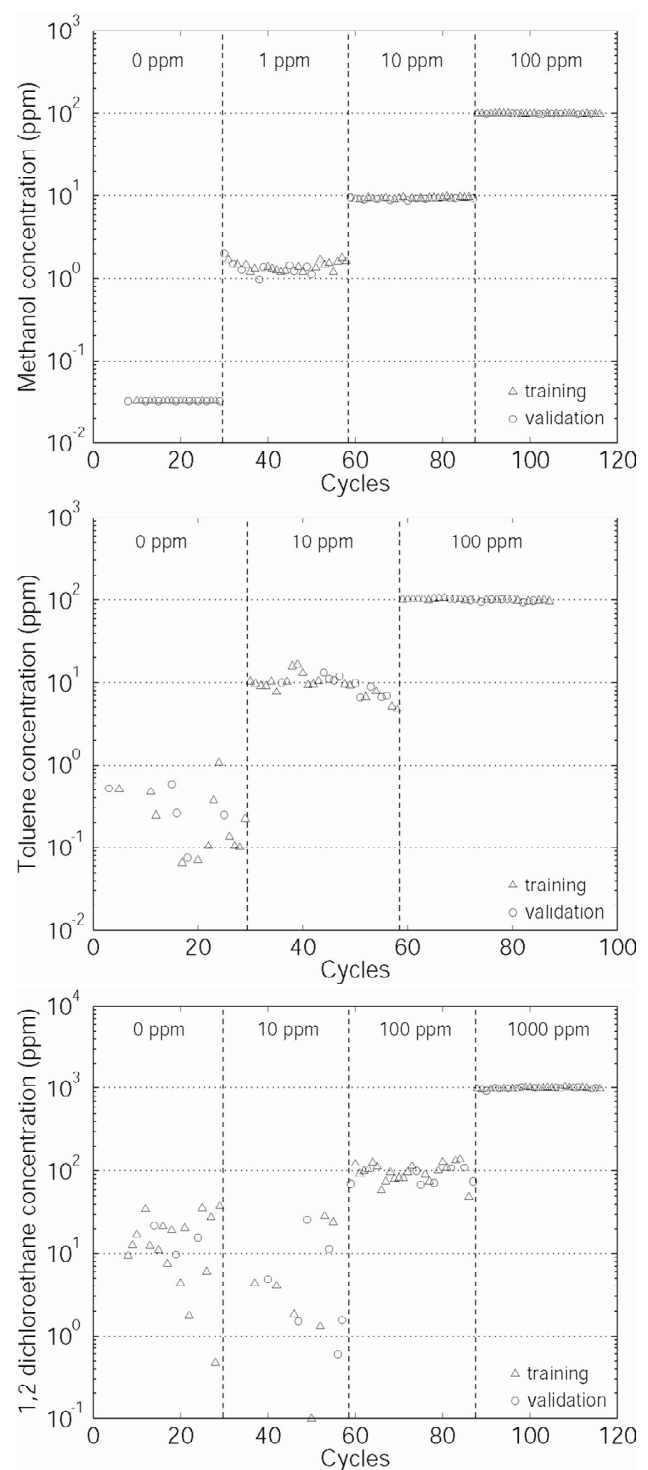

Figure 5. Experimental measurements of VOC concentration by ANN for methanol, toluene, and 1,2-dichloroethane.

\section{CONCLUSIONS}

The demonstrated water monitoring platform combines a silicon-based microhotplate sensor chip capable of conductometric measurement of organic solvents in the gas phase with a microfluidic 2-phase flow network for effective solvent extraction. This study has primarily focused on evaluating the sensitivity of the system to a suite of VOCs diluted in water, with results indicating approximate detection limits of $1 \mathrm{ppm}$ for methanol, 10 ppm for toluene, and $100 \mathrm{ppm}$ for 1,2-dichloroethane. Although the sensitivity is lower than that required for effective monitoring of drinking water supplies, the system in its current form could be amenable to monitoring point source contaminant emissions where higher concentrations are present.

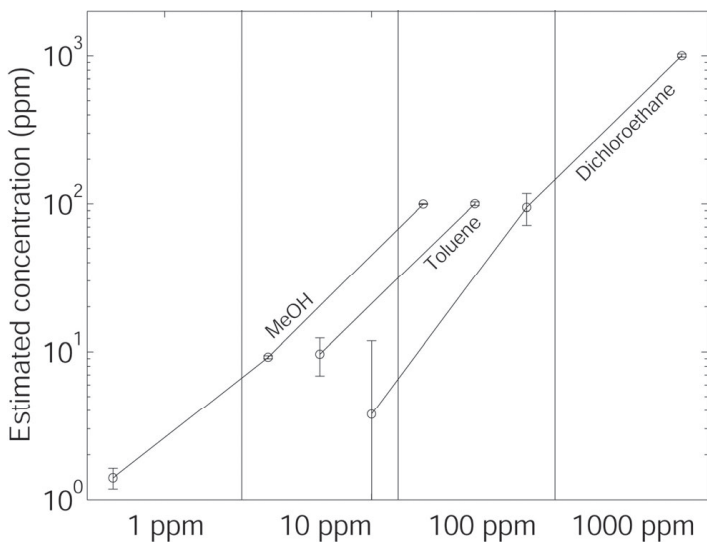

Figure 6. Summary of sensor performance for each VOC analyte. Error bars indicate standard deviations for the ANN data provided in Figure 5.

\section{REFERENCES}

[1] A. Szczurek, P. M. Szecowka, B. W. Licznerski, Sensors and Actuators B, 58 (1999), pp. 427-432.

[2] S. D. Richardson, Anal. Chem., 73 (2001), pp. 2719-2734.

[3] C. K. Ho, M. T. Itamura, M. Kelley, R. C. Hughes, Review of chemical sensors for in-situ monitoring of volatile contaminants, SAND2001-0643, Sandia National Laboratories, Albuquerque, N.M (2001).

[4] C. K. Ho, R. C. Hughes, Sensors, 2 (2002), pp. 23-34.

[5] C. K. Ho, E. R. Lindgren, K. S. Rawlinson, L. K. McGrath, J. L. Wright, Sensors, 3 (2003), pp. 236-247.

[6] C. Lu, W. Tian, W. H. Steinecker, A. Guyon, M. Agah, M. C. Oborny, R. D. Sacks, K. D. Wise, S. W. Pang, E.T. Zellers, Proc. Micro Total Analysis Systems, Squaw Valley, CA (2003), pp. 411-414,.

[7] M. Tokeshi, T. Minagawa, T. Kitamori, Anal. Chem., 72 (2000), pp. 1711-1714.

[8] S. Semancik, R. E. Cavicchi, M. C. Wheeler, J. E. Tiffany, G. E. Poirier, R. M. Walton, J. S. Suehle, B. Panchapakesan, D. L. DeVoe, Sensors and Actuators B, 77 (2001), pp. 579-591.

[9] W. E. TeGrotenhuis, R. J. Cameron, M. G. Butcher, P. M. Martin, R. S. Wegeng, Separation Science and Technology, 34 (1999), pp. 951-974.

[10] Z. Guo, N. F. Roache, Annals of Occupational Hygiene, 47 (2003), pp. 279-286.

[11] C. L. Yaws, Handbook of transport property data: viscosity, thermal conductivity, and diffusion coefficients of liquids and gases, Gulf Publishing, Houston, TX, 1995.

[12] R. J. Millington, Science, 130 (1959), pp. 100-102.

[13] R. E. Cavicchi, J. S. Suehle, K. G. Kreider, M. Gaitan, P. Chaparala, IEEE Electron Device Letters, 16 (1995), pp. 286288.

[14] S. Semancik, R. Cavicchi, Accounts of Chemical Research, 31 (1998), pp. 279-287.

[15] Z. Boger, D. C. Meier, R. E. Cavicchi, S. Semancik, Sensor Letters, 1 (2003), pp. 86-92.

\begin{tabular}{|l|c|c||c|c||c|c||c|c||}
\multicolumn{1}{l||}{} & \multicolumn{2}{c||}{$1 \mathrm{ppm}$} & \multicolumn{2}{c||}{$10 \mathrm{ppm}$} & \multicolumn{2}{c||}{$100 \mathrm{ppm}$} & \multicolumn{2}{c||}{$1000 \mathrm{ppm}$} \\
\hline & $\Delta$ & $\sigma$ & $\Delta$ & $\sigma$ & $\Delta$ & $\sigma$ & $\Delta$ & $\sigma$ \\
\hline Methanol & $40.1 \%$ & $15.7 \%$ & $-8.0 \%$ & $2.4 \%$ & $-0.4 \%$ & $0.6 \%$ & $\mathrm{n} / \mathrm{a}$ & $\mathrm{n} / \mathrm{a}$ \\
\hline Toluene & $\mathrm{n} / \mathrm{a}$ & $\mathrm{n} / \mathrm{a}$ & $-3.6 \%$ & $28.5 \%$ & $0.3 \%$ & $2.9 \%$ & $\mathrm{n} / \mathrm{a}$ & $\mathrm{n} / \mathrm{a}$ \\
\hline 1,2-dichloroethane & $\mathrm{n} / \mathrm{a}$ & $\mathrm{n} / \mathrm{a}$ & $-62.2 \%$ & $214 \%$ & $05.4 \%$ & $24.2 \%$ & $0.2 \%$ & $2.4 \%$ \\
\hline
\end{tabular}

Table 1. Summary of estimated concentration average error ( $\Delta)$ and standard deviation $(\sigma)$ relative to the known concentration. 